\title{
Preconditioned HLLC scheme for incompressible viscous flow simulation
}

\author{
Z. Qian ${ }^{1,2} \&$ C.-H. Lee ${ }^{3}$ \\ ${ }^{1}$ AVIC Aerodynamics Research Institute, China \\ ${ }^{2}$ AVIC Aeronautical Science and Technology Key Lab for High Speed \\ and High Reynolds Number Aerodynamic Force Research, China \\ ${ }^{3}$ National Laboratory for Computational Fluid Dynamics, \\ School of Aeronautic Science and Engineering, Beihang University, \\ China
}

\begin{abstract}
A new HLLC approximate Riemann solver with preconditioning technique based on the pseudo-compressibility formulation for numerical simulation of the incompressible viscous flows has been proposed, which follows the HLLC Riemann solver (Harten, Lax and van Leer solver with contact resolution modified by Toro) for the compressible flow system. In the authors' previous work, the preconditioned Roe's Riemann solver was applied to the finite difference discretization of the inviscid flux. Although the Roe's Riemann solver is found to be an accurate and robust scheme in various numerical computations, the HLLC Riemann solver is more suitable for the pseudo-compressible NavierStokes equations, in which the inviscid flux vector is a non-homogeneous function of degree one of the flow field vector; however the Roe's solver is restricted to the homogeneous problems. Numerical investigations have been performed in order to demonstrate the efficiency and accuracy of the present procedure. The present results are found to be good agreement with the exact solutions, existing numerical results or experimental data.

Keywords: precondition, HLLC scheme, pseudo-compressibility, incompressible viscous flows, LU-SGS.
\end{abstract}

\section{Introduction}

The difficulty of numerically solving the incompressible Navier-Stokes (N-S) equations is the decoupling of the velocity and pressure fields while the zero 
divergence condition is hard to be satisfied. The pseudo-compressibility method proposed by Chorin [1] introduced an additional time derivative of the pressure field to the continuity equation, and so the continuity and momentum equations are solved in a coupling manner.

The precondition concept applied on the incompressible flow simulation was proposed by Turkel [2], then the authors [3] further developed this procedure and applied it to numerical simulation of the pseudo-compressible N-S equations. The influences of precondition parameter and pseudo-compressibility factors on the convergence rate were also investigated systematically, and the optimal parameters were identified in the author's previous paper [3].

Although the pseudo-compressibility method has been widely used for about three decades [4-7], only two classes of scheme, namely the Jameson's central scheme [8] and the Roe's upwind scheme [9], are employed for the inviscid flux terms. The author's previous work [3] shown that the Roe's upwind scheme is superior to the Jameson's central scheme for lower dissipation and free of turned parameter. However, since the inviscid flux vector $F$ of the pseudo-compressible $\mathrm{N}-\mathrm{S}$ equations is not homogeneous function of degree one of the flow field vector $U$, namely $\Delta F=A \Delta U$, and also the homogeneous property is a critical restriction in Roe's solver, consequently Roe's upwind scheme may be not proper in the pseudo-compressibility case.

In the present paper, a new approximate Riemann solver for upwind scheme for incompressible flow is derived following the HLL scheme propose by Harten et al. [10] and its modified form HLLC scheme proposed by Toro et al. [11] for compressible flow simulations. In this approximate Riemann solver we do not assume the homogeneous property of the inviscid flux, so the proposed approximate Riemann solver is more proper and general for incompressible flow simulation.

A numerical procedure using both the proposed HLLC upwind finite difference scheme and the precondition technology is then established for the viscous incompressible flow simulation. Several numerical test cases, including low Reynolds number flow over a circular cylinder, laminar flow over a flat plate, and high Reynolds turbulent flow over the S809 airfoil with the two equations k- $\omega$ SST turbulent model, are numerically investigated in this paper in order to demonstrate the efficiency and accuracy of the present procedure. The present results are found to be good agreement with the exact solutions, existing numerical results or experimental data.

\section{Preconditioning for pseudo-compressibility Navier-Stokes equations}

The pseudo-compressibility procedure and the preconditioning technique for convergence acceleration for stiff hyperbolic equations are employed to develop a highly efficient in-house code for solving two dimensional incompressible flows numerically in the author's previous work [3]. 
The preconditioned pseudo-compressibility Navier-Stokes equations in Cartesian coordinates can be given as below

$$
\Gamma^{-1} Q_{t}+\left(E-E^{v}\right)_{x}+\left(F-F^{v}\right)_{y}=0,
$$

where

$$
\Gamma^{-1}=\left(\begin{array}{ccc}
1 / \beta^{2} & 0 & 0 \\
\alpha u / \beta^{2} & 1 & 0 \\
\alpha v / \beta^{2} & 0 & 1
\end{array}\right),
$$

The state variables $Q$ and fluxes $E, F, E^{v}, F^{v}$ are identical to those given in [3], where $\beta$ denotes the pseudo-compressibility factor, and $\alpha$ denotes the parameter introduced by precondition. The selection of parameters $\alpha$ and $\beta$ is critical to the accuracy and convergence rate, and some detailed discussions are already given in [3].

\section{Numerical schemes}

The semi-discretized finite difference method is used in the present paper. First, the spatial derivatives are discretized while the time variation remains continuous. Then, the time marching in temporal can be solved by the implicit LU-SGS (Lower-Upper Symmetric Gauss-Seidel) algorithm.

\subsection{Spatial scheme}

A new approximate Riemann solver for incompressible flow is derived following the HLL scheme propose by Harten, Lax and van Leer and its modified form HLLC scheme proposed by Toro, Spruce and Speares for compressible flow simulations.

\subsubsection{The HLL approximate Riemann solver for incompressible flow}

Harten et al. [10],Toro [12] put forward the following approximate Riemann solver

$$
\hat{U}(x, t)=\left\{\begin{array}{lll}
U_{L}, & \text { if } & x / t<S_{L}, \\
U_{\text {hll }}, & \text { if } & S_{L} \leq x / t \leq S_{R}, \\
U_{R}, & \text { if } & x / t>S_{R} .
\end{array}\right.
$$

where is $U_{h l l}$ is the integral average of the exact solution of the Riemann problem between the slowest and fastest waves, $S_{L}$ and $S_{R}$ are the fastest wave velocities perturbing the initial data states $U_{L}$ and $U_{R}$ respectively. Figure 1 shows the structure of this approximate solution of the Riemann problem. 


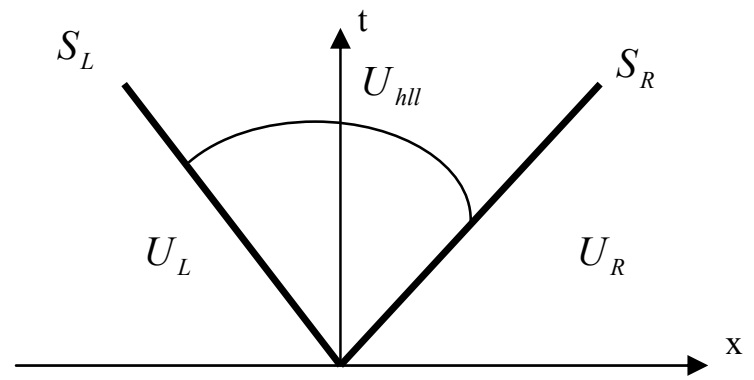

Figure 1: Approximate HLL Riemann solver.

Provided that the signal speeds $S_{L}$ and $S_{R}$ are known, $U_{h l l}$ can be given by

$$
\mathrm{U}_{\mathrm{hll}}=\frac{S_{R} U_{R}-S_{L} U_{L}+F_{L}-F_{R}}{S_{R}-S_{L}}
$$

following Harten, Lax and van Leer's formula. The corresponding flux at the cell interface for the HLL scheme is then given by

$$
F_{i+1 / 2}^{h l l}= \begin{cases}\frac{F_{L},}{S_{R} F_{L}-S_{L} F_{R}+S_{L} S_{R}\left(U_{R}-U_{L}\right)} & \text { if } 0<S_{L}, \\ S_{R}-S_{L}, & \text { if } \quad S_{L} \leq 0 \leq S_{R}, \\ F_{R}, & \text { if } 0>S_{R} .\end{cases}
$$

Given an algorithm to compute the speeds $S_{L}$ and $S_{R}$ one can have an approximate intercell flux. Procedures to estimate the wave speeds $S_{L}$ and $S_{R}$ in this paper are given by

$$
S_{L}=\min \left\{u_{L}-a_{L}, u_{R}-a_{R}\right\}, S_{R}=\max \left\{u_{L}+a_{L}, u_{R}+a_{R}\right\} .
$$

Following Harten, Lax and van Leer, the HLL scheme for the pseudocompressibility Navier-Stokes equations can be derived. The artificial speed of sound is given by

$$
a_{L, R}=\sqrt{u_{L, R}^{2}+\beta^{2}}
$$

For incompressible flow the density is assumed to be constant and the energy equation is decoupled from the continuity and momentum equations. So the intermediate pressure $p_{*}$ and normal velocity $u_{*}$ can be obtained from the Rankine-Hugoniot Conditions across the two wave system similar to the original HLL scheme for compressible flow, which is given as 


$$
\begin{gathered}
u_{*}=\frac{S_{R} u_{R}-S_{L} u_{L}+u_{L}^{2}+P_{L}-u_{R}^{2}-P_{R}}{S_{R}-S_{L}}, \\
p_{*}=\frac{S_{R} p_{R}-S_{L} p_{L}+\beta^{2} u_{L}-\beta^{2} u_{R}}{S_{R}-S_{L}}
\end{gathered}
$$

Also for incompressible flow, the two wave speeds are always satisfied that

$$
u-a<0, u+a>0 \text {. }
$$

This means that the two approximate wave speeds $S_{L}$ and $S_{R}$ are always satisfied that

$$
S_{L}<0, S_{R}>0,
$$

consequently the corresponding cell interface flux of the HLL scheme for incompressible can be simplified as

$$
F_{i+1 / 2}^{\text {hll }}=\frac{S_{R} F_{L}-S_{L} F_{R}+S_{L} S_{R}\left(U_{R}-U_{L}\right)}{S_{R}-S_{L}} .
$$

Note that this Riemann solver consists of just three constant states separated by two waves. All intermediate states separated by intermediate waves are lumped into the single $U_{h l l}$ state without regard for the spatial variations of the solution of the Riemann problem in the Star region, so a shortcoming of the HLL scheme, with too serious dissipation, is exposed.

\subsubsection{The HLLC approximate Riemann solver for incompressible flow}

As pointed out by Harten et al. themselves [10], the too much dissipation of the HLL Riemann solver may be alleviated by restoring the missing waves. Accordingly, Toro et al. $[11,12]$ proposed the so called HLLC scheme, where C stands for Contact. In the HLLC scheme the missing middle waves are put back into the structure of the approximate Riemann solver.

Consider Figure 2, in which the complete structure of the solution of the Riemann problem is depicted. As shown in the figure, in addition to the slowest and fastest signal speeds $S_{L}$ and $S_{R}$, we include a middle wave of speed $S_{*}$, corresponding to the shear wave with eigenvalue $u$.

Following Toro et al. [11], the HLLC flux can be written as

$$
F_{i+1 / 2}^{\text {hllc }}= \begin{cases}F_{L}, & \text { if } 0<S_{L}, \\ F_{*_{L}}=F_{L}+S_{L}\left(U_{*_{L}}-U_{L}\right), & \text { if } \quad S_{L} \leq 0<S_{*}, \\ F_{*_{R}}=F_{R}+S_{R}\left(U_{*_{R}}-U_{L}\right), & \text { if } \quad S_{*} \leq 0 \leq S_{R}, \\ F_{R}, & \text { if } 0>S_{R} .\end{cases}
$$




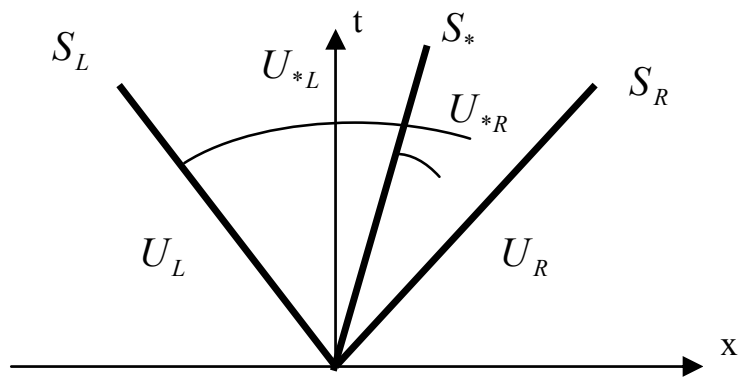

Figure 2: $\quad$ Approximate HLLC Riemann solver.

where $U_{*_{L}}$ and $U_{*_{R}}$ denote to the integral averages between the shear wave and the left and right waves respectively, which can be given by the following Rankine-Hugoniot conditions across each of the waves of speeds $S_{L}, S_{*}$ and $S_{R}$.

$$
\begin{gathered}
F_{*_{L}}=F_{L}+S_{L}\left(U_{*_{L}}-U_{L}\right), \\
F_{*_{R}}=F_{*_{L}}+S_{*}\left(U_{*_{R}}-U_{*_{L}}\right), \\
F_{*_{R}}=F_{R}+S_{R}\left(U_{*_{R}}-U_{R}\right) .
\end{gathered}
$$

Here condition (14)-(16) are three equations for the four unknowns vectors $U_{*_{L}}$, $U_{*_{R}}, F_{*_{L}}$ and $F_{*_{R}}$. The aim is to find the vectors $U_{*_{L}}$ and $U_{*_{R}}$, so that the fluxes $F_{*_{L}}$ and $F_{*_{R}}$ can be determined from (14) and (16) respectively. For incompressible flow the following conditions are must guaranteed.

$$
u_{*_{L}}=u_{*_{R}}=u_{*}, p_{*_{L}}=p_{*_{R}}=p_{*}, v_{*_{L}}=v_{L}, v_{*_{R}}=v_{R} \text {. }
$$

Consequently, we can obtain that $u_{*}$ and $p_{*}$ are the same as equation (8) and (9) respectively, and $v_{*_{L}}=v_{L}, v_{*_{R}}=v_{R}, S_{*}=u_{*}$. Since $S_{L}<0$ and $S_{R}>0$, the corresponding intercell flux of the HLLC scheme for incompressible can be simplified as

$$
F_{i+1 / 2}^{\text {hllc }}=\left\{\begin{array}{lll}
F_{L^{*}}=F_{L}+S_{L}\left(U_{*_{L}}-U_{L}\right), & \text { if } & 0 \leq S_{*}, \\
F_{R^{*}}=F_{R}+S_{R}\left(U_{* R}-U_{L}\right), & \text { if } & S_{*}<0 .
\end{array}\right.
$$

\subsubsection{Second order accurate interface values $U_{L}$ and $U_{R}$}

In order to promote the accuracy, we employ the second order MUSCL scheme [13] in the present study. Accordingly, the left and right states are given respectively by 


$$
\left.\begin{array}{l}
U_{L}=U_{i}+\varphi_{L} \Delta U_{i}, \\
U_{R}=U_{i+1}-\varphi_{R} \Delta U_{i+1},
\end{array}\right\}
$$

in which $\Delta U_{i}=U_{i}-U_{i-1}, \varphi$ denotes to the limiter function. In the present paper, the minmod limiter is used unless specified.

\subsubsection{Viscous term computations and numerical boundary conditions}

The viscous terms of eq. (1) are discretized by the second-order central difference. The boundary conditions imposed on the solid wall are the no-slip condition for viscous flows, and the pressure on the wall is evaluated by extrapolation from the interior points. For the far field boundary, the conditions are specified according to the direction of the in-flow or out-flow, where the velocities are given, and the pressure is extrapolated from the interior nodes at the inflow boundary; whereas the pressure is given, and the velocities are extrapolated from the interior nodes at the outflow boundary.

\subsection{Time advancement}

The LU-SGS (Lower-Upper Symmetric Gauss-Seidel) formulation proposed by Yoon and Jameson [14] is used, which is one of the most commonly used implicit methods for compressible flows simulations. We generalize the formulation to the preconditioned pseudo-compressible Navier-Stokes equations, and details can be found in the authors' previous work [3].

\section{Numerical results}

Some validating tests are presented in this section, including low Reynolds number flow over a circular cylinder, laminar flow over a flat plate, and high Reynolds turbulent flow over the S809 airfoil with the two equations k- $\omega$ SST turbulence model. These cases are chosen for comparisons with exact analytic solutions or existing experimental data.

\subsection{Low Reynolds number flow over circular cylinder}

The low Reynolds number incompressible flow over a circular cylinder is a benchmark problem for incompressible flows, for which many experimental and computational results can be utilized for comparison $[4,15,16]$. In this section, the problem is simulated by the algorithm proposed in previous sections.

The characteristics of the flow field are sensitive to the Reynolds number. The case of $\mathrm{Re}=40$ is computed, and the flow renders as a pair of symmetric separated vortices. The mesh used in this paper consists of a single gird zone with an $\mathrm{O}$ type topology, and is composed of $65 \times 181$ in the streamwise and wall normal direction, respectively, with the first grid spacing of 0.005 diameters and stretching to the outer boundary of 20 diameters in the normal direction. 
The streamlines of the flow at $\mathrm{Re}=40$ computed by the present scheme are depicted in Figure 3. The separation length measured from the rear stagnation point of the cylinder in cylinder diameters, the separation angle which defines the point of separation from the body, and the drag coefficient in each case are also shown in Table 1.They are consistent with the experimental data $[15,16]$ and the computational results listed in [4]. The errors of the separation length and separation angle relative to [16] are $2.35 \%$ and $0.37 \%$ respectively, and that of the drag coefficient relative to [15] is $2.42 \%$. Moreover, all the errors of the numerical results relative to the experimental data are less than $5 \%$, which demonstrate the reliability of the present method.

Table 1: Comparisons with experimental and other numerical results at $\operatorname{Re}=40$.

\begin{tabular}{cccc}
\hline & $\begin{array}{c}\text { Length of } \\
\text { separation (L/D) } \\
\text { (Errors relative to } \\
[16])\end{array}$ & $\begin{array}{c}\text { Angle of } \\
\text { separation }\left({ }^{\circ}\right) \\
(\text { Errors relative to } \\
[16])\end{array}$ & $\begin{array}{c}\text { Drag coefficient } \\
\text { Errors relative to } \\
[15])\end{array}$ \\
\hline Present & $2.08(-2.35 \%)$ & $53.3(-0.37 \%)$ & $1.610(-2.42 \%)$ \\
$\begin{array}{c}\text { Reference [4] } \\
\text { (Numerical) } \\
\text { Reference } \\
{[15]}\end{array}$ & $2.29(+7.51 \%)$ & $53.0(-0.93 \%)$ & $1.549(-6.12 \%)$ \\
$\begin{array}{c}\text { (experimental) } \\
\text { Reference } \\
{[16]}\end{array}$ & - & - & \\
(experimental) & 2.13 & & 1.65 \\
\hline
\end{tabular}

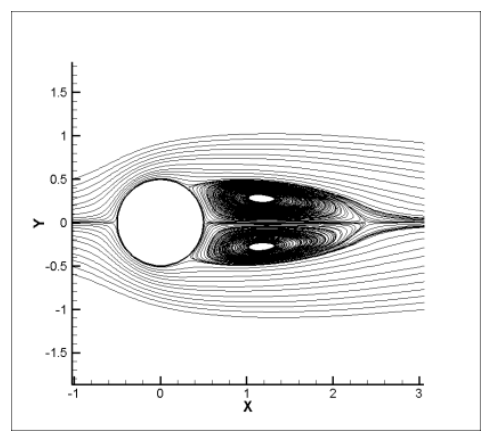

Figure 3: $\quad$ The streamlines at $\mathrm{Re}=40$. 


\subsection{Laminar flow past flat plate}

The incompressible laminar flow over a flat plate is commonly called a Blasius flow with an analytical similarity solution. This test case is used to validate the capability of the presently developed procedure for boundary layer flow simulation. The mesh is composed of $109 \times 50$ grids in the stream wise and wall normal direction, respectively, clustered at the leading and trailing edges of the flat plate. The computational region is stretched to the upper and upwind boundary of 5 times the length of the plate, and to the out flow boundary with 6 times the length. The Reynolds number defined by the length of the plate is $1.0 \times 10^{5}$. The grid is clustered near the wall due to great gradient in the boundary layer.

The velocity profile computed by the present scheme is plotted in Figure 4 together with the Blasius solution, where the abscissa $y^{\prime}=y \sqrt{\operatorname{Re}_{x}} / x$, and the ordinate is the dimensionless velocity $u$. It is found that the present preconditioned HLLC scheme is capable of providing numerical solutions well consistent with the Blasius solution.

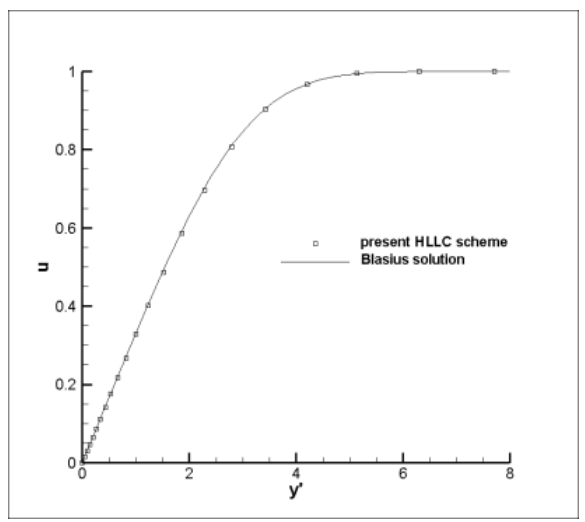

Figure 4: The velocity profiles of the laminar flat plate.

\subsection{High Reynolds turbulent flow around $\mathrm{S809}$ airfoil}

The S809 airfoil was designed specially for wind turbine applications, the thickness ratio of which is $21 \%$. A $0.6 \mathrm{~m}$ chord length model of this airfoil has been tested in a $1.8 \mathrm{~m} \times 2.5 \mathrm{~m}$ low turbulence wind tunnel [17]. In this numerical test, the Reynolds number is $2.0 \times 10^{6}$ and the angle of attack is $9.22^{\circ}$. It shows the reliability of the proposed algorithm for turbulent flow simulation.

The mesh as shown in Figure 5 consists of a single gird zone with an O type topology, and is composed of $307 \times 120$ in the stream wise and wall normal direction, respectively, with the first grid spacing of $1.0 \times 10^{-5}$ chord length and stretching to the outer boundary of 20 diameters. The Menter's $k-\omega S S T$ turbulence model [18], which takes the advantages of the $k-\varepsilon$ model [19] and 
Wilcox's $k-\omega$ model [20], is used. This model excludes the sensitivity to the inflow turbulence intensity and retains the numerical stability near the wall.

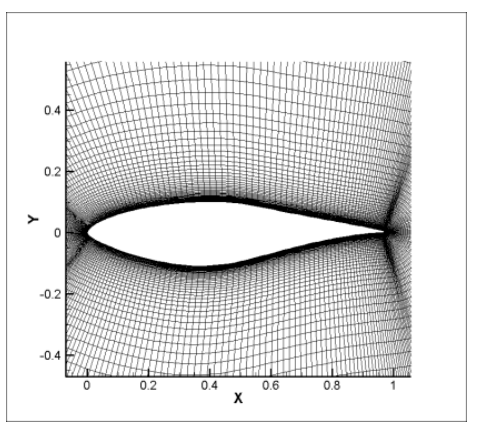

Figure 5: $\quad$ Mesh for S809 airfoil.

The computed contours of pressure, turbulence viscosity ratio and turbulence kinetic intensity are shown in Figure 6. Figure 7 depicts the comparison of the computed wall pressure coefficient and the experimental test data [17], and good agreement is shown.
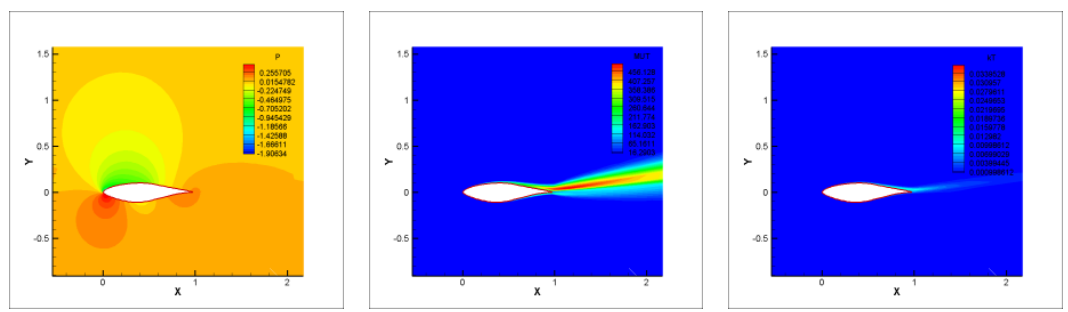

(a) Pressure (b) Turbulence viscosity ratio (c) Turbulence kinetic energy

Figure 6: The contours of pressure, turbulence viscosity ratio and turbulence kinetic intensity.

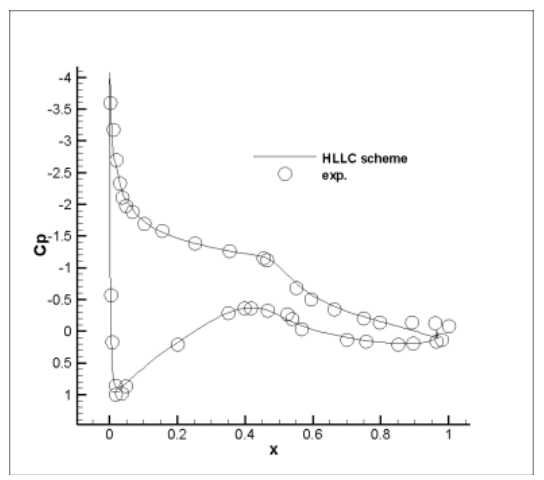

Figure 7: Comparison of the computed and experimental wall pressure coefficients. 


\section{Conclusions}

This paper derives the preconditioned HLLC finite difference scheme for the incompressible flow by virtue of the pseudo-compressibility procedure. The proposed scheme does not assume the homogeneous property of the inviscid flux, so the proposed approximate Riemann solver is more proper and general for incompressible flow simulation. The preconditioned technique is used to accelerate the time marching, and the implicit LU-SGS time integration method is adopted to integrate temporally. The reliability of the present procedure are demonstrated by applications to low Reynolds number flow over a circular cylinder, laminar flow over a flat plate, and high Reynolds turbulent flow over the S809 airfoil. It is found that the numerical results of the present algorithm are in good agreement with theoretical solutions or experimental data.

\section{References}

[1] Chorin A J. A numerical method for solving incompressible viscous flow problems. J. Comput. Phys., 2: 12-26, 1967.

[2] Turkel E. Preconditioning methods for solving the incompressible and low speed compressible equations. J. Comput. Phys., 72: 227-298, 1987.

[3] Qian Zhansen, Zhang Jingbai, Li Chunxuan. Preconditioned pseudocompressibility methods for incompressible Navier-Stokes equations. SCIENCE CHINA Phys. Mech. Astronomy., 53(11): 2090-2102, 2010.

[4] Rogers S E, Kwak D. An Upwind-Differencing Scheme for the Incompressible Navier-Stokes Equations. NASA TM-101051, 1988.

[5] Rogers S E, Kwak D. Upwind differencing scheme for the time-accurate incompressible Navier-Stokes equations. AIAA Journal, 28: 253-262, 1990.

[6] Kwak D, Chakravarthy S R. A three-dimensional incompressible NavierStokes flow solver using primitive variables. AIAA Journal, 24: 390-396, 1986.

[7] Tamamidis P, Zhang G, Assanis D N. Comparison of pressure-based and artificial compressibility methods for solving 3D steady incompressible viscous flows. J Computational Physics, 124: 1-13, 1996.

[8] Jameson A. Time dependent calculations using multigrid with application to unsteady flows past airfoils and wings. AIAA Paper 91-1596, 1991.

[9] Roe P L. Approximate Riemann Solvers, parameter vectors and difference schemes. J. Comput. Phys., 43: 357-372, 1981.

[10] Harten A, Lax P D, van Leer B. On upstream differencing and Godunovtype schemes for hyperbolic conservation laws. SIAM Review, 25(1): 35 61, 1983.

[11] Toro E F, Spruce M, Speares W. Restoration of the contact surface in the HLL-Riemann solver. Shock Waves, 4: 25-34, 1994.

[12] E.F.Toro. Riemann solvers and numerical methods for fluid dynamics: A practical introduction. Springer, 1997. 
[13] van Leer B. Towards the ultimate conservation difference scheme:V. A second-order sequel to Godunov's method. Journal of Computational Physics, 32: 101-136, 1979.

[14] Yoon S, Jameson A. Lower-upper symmetric-Gauss-Seidel method for the Euler and Navier-Stokes equations. AIAA Journal, 26: 1025-1026, 1988.

[15] Tritton D J. Experiments on the flow past a circular cylinder at low Reynolds numbers. J Fluid Mech, 6: 547-567, 1959.

[16] Contanceau M, Bouard R. Experimental determination of the main features of the viscous flow in the wake of a circular cylinder in uniform translation. Part 1. steady flow. J Fluid Mech, 79: 231- 256, 1972.

[17] Somers D M. Design and experimental results for the S809 airfoil. NREL/SR-440-6918, 1997.

[18] Menter F. Zonal two equation k-w turbulence models for aerodynamic flows. AIAA Paper 93-2906, 1993.

[19] Abid R. Evaluation of two-equation turbulence models for predicting transitional flows. International journal of Engineering Science, 131: 831840, 1993.

[20] Wilcox D. Turbulence modeling for CFD. DCW Industries, Inc., La Canada, California, 1993. 